\title{
Exercise for slimming
}

\author{
BY J. F. ANDREWS \\ Department of Physiology, Trinity College Dublin, Dublin 2, Republic of Ireland
}

The pejorative aphorism Obesity is the result of greed and sloth has come to be applied to the fat individual. The inferences implied are widely accepted not only by the public and journalists but by the health-care professional and the nutritional scientist too. Is this justified particularly with respect to sloth?

Of course, fat deposition must be the result of excess intake of energy in relation to metabolic requirements (see Fig. 1), integrated over time and persisting for a long period of time, but it is not necessarily the result of 'greed' or of 'sloth' (Stern, 1984). Equally
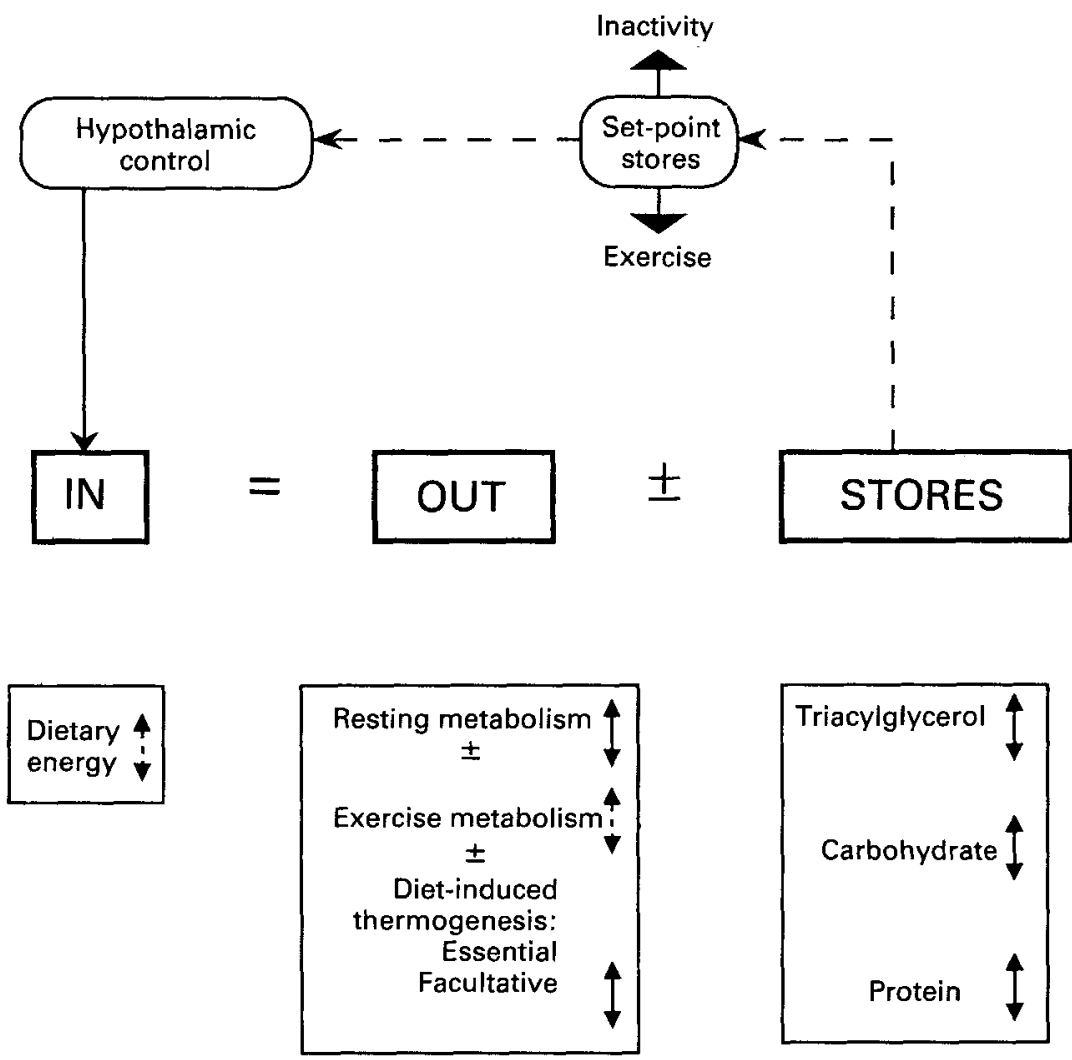

Fig. 1. The energy balance equation. The central set-point mechanism for regulation of stored energy, its modulation by exercise training, and the sensory input indicating state of energy storage, remain hypothetical. $\downarrow$. $\downarrow$ Regulated variables: $\downarrow$. the control is autonomic: $\downarrow$ there is a voluntary element. The diagram shows that increasing exercise must draw on energy stores when dietary intake is reduced. It does not indicate the temporal and quantitative elements which make this a small proportion of fat stores in the obese. 
loss of fat from stores can only be achieved if there is a net energy deficit, also integrated over time and sustained too for a long period.

'Dieting' must be the major plank of any weight reduction or 'slimming' programme (for quantitative justification see below). But what of exercise? Can increased exercise, a reversal of the 'sloth' supposedly contributing to the development of obesity, contribute significantly to weight loss? The present review will examine the evidence bearing on this question, giving emphasis to a quantitative analysis of the energetics of exercise in the obese. I conclude that the weight loss benefits of exercise have been overemphasized, though the benefits to general health and well-being must be stressed.

\section{DOES EXERCISE CONTRIBUTE TO WEIGHT LOSS?}

A considerable number of studies have been done to investigate this question, with very variable results with respect to the effectiveness of exercise for weight loss. This is perhaps not surprising considering the vagueness of the word 'exercise' and the almost infinite possibility of regimens which could fall under the heading of a paper entitled The effect of exercise on weight loss in the obese. An additional variable, affecting the results of these studies, concerns the input side of the energy balance equation, dietary energy intake, and whether or not in any study intake is either deliberately reduced, held constant or allowed to free-run with the possibility of regulated compensatory increase (Fig. 1).

Space does not allow for an individual examination of these historic studies, but a number of recent reviews of them are available giving a comprehensive list of references. Epstein \& Wing (1980) reviewed studies in which the effect of exercise alone on body-weight was examined. From the sixteen studies they surveyed a mean weight loss was calculated at $-0.05 \mathrm{~kg} / \mathrm{week}$, a very modest rate compared with that possible from energy restriction. In a more recent paper, Stern et al. (1989) overviewing several reviews published in the preceding decade (Wing \& Jeffrey, 1979; Björntorp, 1982; Wilmore, 1983; Stern, 1984; Tremblay et al. 1985) concurred with Epstein \& Wing (1980) that exercise had only modest effects, by an order of magnitude less, on rate of weight loss, compared with the possibilities resulting from the restriction of energy intake alone. Therefore, calculating the long hours of exercise required to lose a significant proportion of excess fat stores, many workers, myself included, have expressed pessimism about the weight loss benefits of exercise per se. Katch \& McArdle (1988) have taken the opposite position, however. They consider that over time small incremental benefits are very effective and that the smallness has been much overemphasized: on p. 171 of their book Nutrition, Weight Control and Exercise they refer to what they call 'Misconception Two'. Under this head they emphasize that energy consumed is energy consumed, whatever the time scale. To quote one of their examples: $20 \mathrm{~h}$ of playing golf will require the oxidation of $1 \mathrm{lb}$ of fat. Of course no one will or can play $20 \mathrm{~h}$ of continuous golf. However, one round of $2 \mathrm{~h}$ week over 10 weeks will achieve the same result (on the uncertain presumption that continuous and discontinuous bouts draw equally on fat stores, my caveat, see p. 463). It is the psychological appreciation of this 10 weeks which is at issue: is this a long or short time to achieve what in a very fat person could well be only $1 \%$ of the desired loss of fat? That very much reflects the psychology of the slimming individual. Put into context a single binge meal could quite easily negate the 10 weeks exercise effort. 
THEORETICAL ANALYSIS

Any exercise programme for the slimmer must be put into the context of what is known of the underlying biochemistry, endocrinology and physiology of man. It is facile to prescribe exercise without further definition and refinement. The most fundamental requirement for safe and effective weight loss in the slimmer is that triacylglycerol stores be depleted: this criterion applies to slimming in general and to exercise-induced slimming in particular.

Any slimming programme must be cognizant of the fact that protein structure, the heart being particularly vulnerable, can be a source of energy through catabolism and gluconeogenesis. Protein catabolism must be avoided or minimized in slimming, fat catabolism maximized: far too little emphasis is given to this criterion. Two biochemical criteria and the hormonal environment necessary to produce them must be considered: first, optimum conditions for release of fatty acids from adipocyte triacylglycerol stores, and second the optimum conditions for the full oxidation of these released fatty acids. The physiological circumstances under which use of fatty acids as a fuel for exercise is maximized are then discussed.

Biochemical and endocrinological considerations: sources of energy for exercise. For safe and effective weight loss, exercise must consume fatty acids released from fat stores. Exercise burning glucose will not achieve long-term weight loss and if the glucose comes from protein, can be dangerous.

Muscle mitochondria are in a general sense omnivorous: by that I mean that they can burn either fatty acids or hexose sugars to produce the ATP necessary to operate the contractile mechanism. What conditions favour the consumption of one or other of these fuels?

Glucose is often considered to be the favoured fuel, but in reality this depends on circumstances. However, if glucose is the fuel it will come from either within the muscle fibre, muscle glycogen stores, or from an exogenous source, in the first instance the blood plasma, but as that is a very small store of glucose molecules, and as it is imperative for the maintenance of integrated CNS function that blood glucose concentration be maintained at quite a high level, this must be replenished from liver glycogen as it is consumed. In temporal terms liver glycogen can only maintain blood glucose levels above the hypoglycaemic threshold for relatively brief periods (at the most up to $24 \mathrm{~h}$ at rest; much less, perhaps as little as $1 \mathrm{~h}$ during sustained exercise). Thereafter, if liver glycogen is not replenished by a carbohydrate meal, blood glucose can only be maintained by gluconeogenesis, mainly from protein. Recent elegant work in vivo has quantified the fluxes of different fuels in and out of fat tissue during absorption and in the post-absorptive state (Frayn et al. 1989), confirming the qualitative description of fat as the main source of energy in starvation to be correct. Such work has yet to be done in exercise to determine the in vivo quantitative release of fatty acids from a fat depot.

Therefore, for maximum weight loss in exercise the first requisite is to ensure the correct hormonal environment to produce the relative high fatty acid:glucose molecules ratio at the muscle mitochondrial membrane which is necessary to favour fatty acid over glucose oxidation. To understand this fully we must first examine the biochemical and hormonal effects occurring at the fat cell during starvation and superimposed exercise. In starvation blood glucose will be low; therefore, insulin low. The hormones of starvation will be high, particularly glucagon. This enhanced glucagon:insulin ratio ensures that glucose transporters are switched off (at the resting muscle fibre too) and that through a 


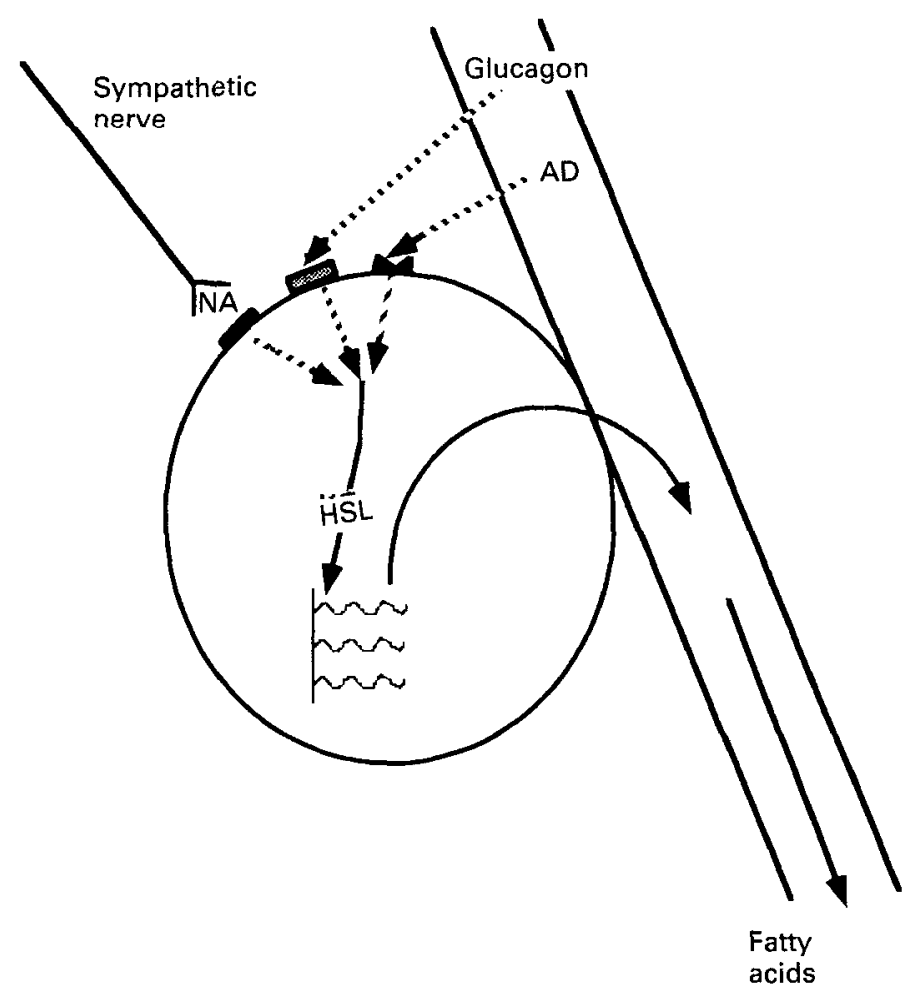

Fig. 2. The white fat cell, site of triacylglycerol (TG) stores, the vast bulk of stored energy in the obese. The diagram shows the cell in the fasted state, during endurance exercise, the physiological conditions best suited to maximize lipolysis by hormone-sensitive lipase (HSL) and, hence, release of fatty acids. Catechols (adrenaline (AD) and noradrenaline (NA)) and glucagon levels are maximized by exercise and starvation respectively, and insulin levels minimized by starvation. This is the hormonal environment which maximizes HSL activity and consequent release of fatty acids. As exercise is ongoing, the working muscles are a sink for the released fatty acids, by their complete oxidation.

cascade of intracellular events hormone sensitive lipase is switched on; therefore lipolysis is occurring, fatty acids are pouring out of the fat cell for oxidation by most tissues of the body, including the muscle fibre (Fig. 2). Fat is sparing glucose. This liberation and subsequent full oxidation of fatty acids is the essential event for weight loss by the obese.

Exercise can enhance this process. First, at the fat cell by further increasing lipolysis when the catechols released on exercise act synergistically with glucagon via separate cell surface receptors, but through a final common pathway to further stimulate hormonesensitive lipase. Second, by providing a major additional oxidative sink for the released fatty acids on top of the resting metabolic requirements of most other tissues (Fig. 2). Thus, requisite one is fulfilled: fatty acids are released and made available, the fatty acids:glucose molecules ratio at muscle mitochondria membrane will have been increased favouring oxidation of the former.

Requisite two concerns the correct physiological environment to ensure that muscular work maximizes oxidation of the fatty acids flowing from the triacylglycerol stores of the fat cell. The rate of consumption of ATP by the working muscle must not be so high that 
the anaerobic threshold be exceeded. At low work rates the increased fatty acid:glucose molecules ratio will ensure their preferential oxidation. If the body, acting through the nervous system calls upon the muscles to provide greater power, this can be done, but at a penalty. Despite the favourable high fatty acids:glucose molecules ratio at the mitochondrial membrane, biochemical and biophysical limitations produce a ratelimiting threshold for the oxidation of fatty acids, possibly concerning fatty acid transfer by the carnitine system into the mitochondria or steps in the $\beta$-oxidation pathway for fatty acid metabolism, or both, particularly at the point of entry into the Krebs cycle where acetyl-CoA may become rate-limiting. To increase rates of ATP production further the fibre must oxidize an increasing proportion of glucose molecules.

Over many years, through determination of respiratory quotient (RQ), much indirect evidence has established the role of fatty acids in providing fuel for whole-body metabolism during exercise (for example, Wolfe et al. 1990). Only a very few historic studies are available to directly measure the fuel mix being taken up by a muscle mass during steady-state exercise at different work rates. One such, at $30 \%$ maximum capacity (Ahlborg et al. 1974), shows fatty acids and glucose contributing 38 and $27 \%$ respectively (molar ratio 1.4:1) to total leg muscle uptake of fuels after $40 \mathrm{~min}$ exercise, with the percentage of fatty acids extracted increasing somewhat as exercise continues (61 and 30 respectively, molar ratio $2: 1$, at $240 \mathrm{~min}$ ). A second study at a much higher work rate of $60 \%$ maximum capacity, but below the anaerobic threshold (Rennie et al. 1976), showed that the fatty acid:glucose uptake molar ratio had fallen very considerably to $0 \cdot 25: 1$. Comparison of studies on exercise energetics is fraught with difficulties: however, these two studies can be taken as positive evidence that proportionally (though not necessarily the absolute level of) fatty acid oxidation falls as power output increases.

At the start of any bout of exercise there is a necessary brief anaerobic phase drawing on glucose stores followed by a phase of high glucose oxidation whilst cardiovascular, respiratory and metabolic adjustments come into play to allow full oxidation of fuel followed by the replacement of glucose fatty acids. In addition it has been shown that at a fixed work rate, with increasing duration of a bout of exercise, the proportion of energy derived from fatty acids steadily increases, this effect continuing for up to $4 \mathrm{~h}$ (Wolfe et al. 1990). Therefore, a single bout of long duration is far more beneficial in terms of fatty acid consumed than the same duration of exercise accumulated through a number of small bouts.

As work rate continues to increase, a level is reached when the full oxidative mechanism becomes saturated and anaerobic metabolism in the cytosol, runaway glycolysis to lactate, energetically inefficient though it may seem, is the only way to provide ATP at the required high rate. Clearly the slimmer should exercise at a rate which maximizes oxidation of fatty acids without exceeding the threshold which requires anaerobic metabolism of glucose. Otherwise the desired benefits of exercise, the diminution of the fat stores by oxidation of the released fatty acids, will not be maximized.

This understanding of the biochemical mechanisms, with the need to maximize fatty acid release from fat stores and in addition to ensure its oxidation, is an essential prerequisite to prescribing effective exercise for weight loss. If fats are simply lipolysed and fatty acids released but not oxidized, the body has no option but to convert them back into stored triacylglycerol again (in the healthy individual the evidence is against ketosis and, thus, ketonuria being a route of energy loss during or after exercise 
(Wallberg-Hendriksson \& Wahren, 1989). This recycling (the so-called fatty-acid cycle) does consume some energy, and this has been the basis for the proposal that such 'futile' cycles are of benefit in weight loss (Newsholme, 1977). However, one cycle of the fatty-acid cycle consumes a rather small proportion of the energy potential of the stored fat, the vast bulk being redeposited in the fat cell. The proponents of this thesis, therefore, suppose that such cycles run free at high rates and, thus, eventually consume much energy. Whole-body studies such as that of Wolfe et al. (1990), show that during exercise the fatty-acid cycle makes little contribution to total metabolism, during recovery, however, recycling does contribute a significant part of post-exercise excess metabolism, albeit that is only a small part of total metabolism (see pp. 464-465). In contrast, Frayn et al. (1989) who investigated fatty acid turnover in the superior adipose tissue of the anterior abdominal wall of man in vivo, could find no evidence for such an extracellular fatty-acid cycle and little evidence for an intracellular fatty-acid cycle. Possibly this was an inappropriate tissue to investigate, since animal work appears to substantiate an intracellular fatty-acid cycle in some adipose tissue depots but not others (Mattocks et al. 1987). The balance of evidence does not support a major role for the fatty-acid cycle in the energetics of man.

Physiology: type and level of exercise. Two criteria must be considered: first, what type and level of exercise maximizes the full oxidation of fatty acids; second, how can this be sustained for a significant duration? Maximizing fatty acid consumption involves exercising below the threshold at which glucose starts replacing fatty acid as fuel, and particularly below the anaerobic threshold. During steady-state exercise the mix of fuel being burnt may be readily determined in the laboratory from the $R Q$, as may also an 'anaerobic' threshold from the onset of blood lactate accumulation (OBLA; Fig. 3).

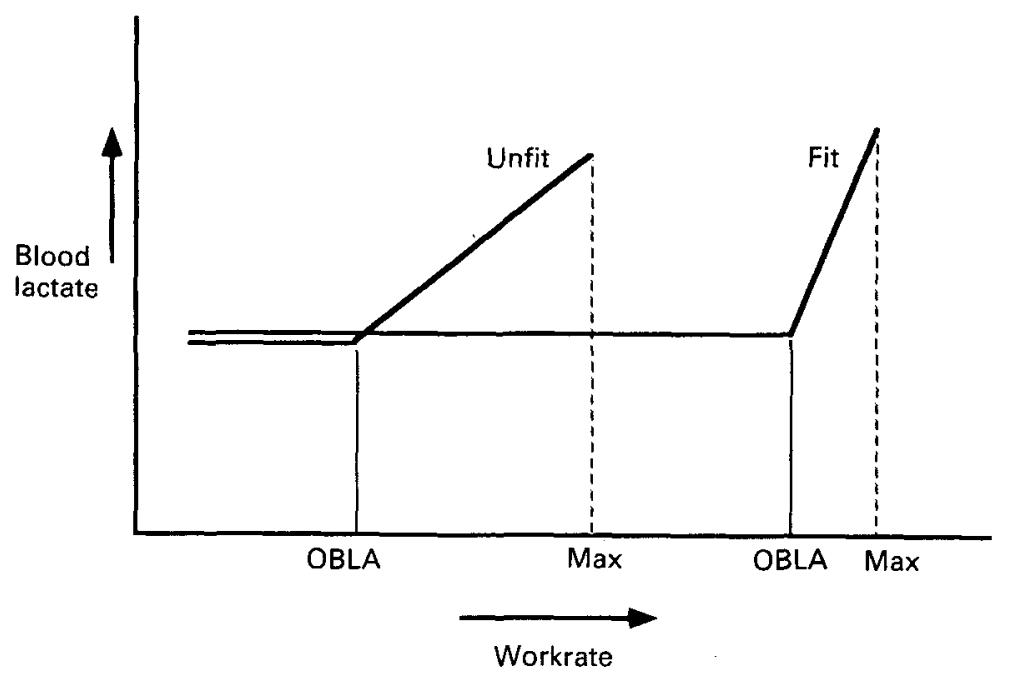

Fig. 3. Aerobic capacity and the effect of training. The onset of blood lactate accumulation (OBLA) is an index of the point at which, as work increases, muscles switch to anaerobic metabolism of glucose. It is not necessarily the point at which fatty acid oxidation is maximized, but is convenient to use in practice. Exercise below OBLA will certainly ensure that much of the oxidized fuel is fatty acid. Note that with training absolute OBLA increases and additionally OBLA as a percentage of maximum capacity increases. Not shown is the increase in endurance time with training. All these factors ensure increasing fatty acid oxidation per exercise bout with training. See Table 1. 
Much experimental experience has led to the following observations: with endurance training the maximum capacity for exercise steadily increases and goes on increasing for a long period of time. At the same time OBLA increases, not just as a proportion of maximum capacity but as an increasing proportion (Fig. 3). What is not evident from the diagram (Fig. 3) is that experience shows that exercise endurance of the individual also increases, particularly at work rates below maximum capacity.

Ideal exercise programme. These physiological observations, linked to the biochemical analysis discussed previously enable the design of an ideal exercise programme for maximizing fatty acid consumption and, therefore, weight loss in the obese. It is presumed that the obese individual is very unfit though this is not necessarily so. Therefore, a training programme of endurance exercise must be instituted which brings about increasing fitness with the benefit of increasing endurance and a gradual increase in the work rate at which OBLA occurs. Thus, slowly and steadily, both rate of fatty acid oxidation and the time-period for which this rate can be sustained, will be increased. To avoid the clash of hormones at the fat-cell receptors this exercise is best done post absorptive when insulin concentrations are at a minimum so that glucagon and catechols can act unopposed, and in consort, to maximize fat-cell lipolysis. Glucose or carbohydrate snacks just before or during the exercise, as correctly indicated for improved performance of champion athletes, are most emphatically counterproductive for the reasons given previously. Equally, 'sustained' exercise is to be preferred to 'on-off' bouts, for the reasons given previously.

If, as will be the case for most slimmers, exercise testing facilities are unavailable to determine OBLA and RQ at different work rates, so as to be able to optimize fatty acid oxidation, the individual will have to listen to the 'wisdom of the body', assessing from internal messages what rate of exercise can be sustained and maintained for many minutes without undue feelings of cardiovascular or respiratory distress.

The health aspects, contraindications and benefits, of exercise are discussed later (pp. $465-470)$.

\section{QUANTITATIVE ANALYSIS}

The energy balance equation (Fig. 1) shows that as exercise is an energy-consuming process, in this sense it must add to the energy deficit of a slimming programme. But is the amount of this energy used in exercise significant. It is my contention that although the amount of energy lost in exercise may be significant in a few not so fat individuals undertaking particularly arduous programmes, that in a general sense, and particularly as degree of obesity increases and presumed degree of fitness decreases reciprocally, each exercise session has only a minor to vanishingly small effect on reducing fat stores: that for the grossly obese the weight loss benefits of exercise have been oversold.

This assertion is justified by the theoretical quantitative analysis of the energy costs of a sustainable bout of exercise which is given in Table 1. Such analyses have been criticized as valueless, particularly the calculation of the number of hours exercise body fat stores would sustain. Of course in a literal sense this is a silly and meaningless calculation, but it is a striking metaphor for the effectiveness of fat as a dense energy store. In Table 1 four model individuals of similar lean body mass but increasing adiposity are compared as to the energy costs of exercise in relation to a sustainable bout at their fitness level: (1) a very fit, very lean, world-class endurance athlete; (2) the 'ideal' $70 \mathrm{~kg}$ young man of normal fitness and clinically desirable adiposity; (3) a moderately 
Table 1. The energy content of the fat depots of four model individuals of identical lean body mass but different adiposity; theoretical analysis of the number of exercise bouts required to reduce adiposity to a clinically desirable 'ideal' value

\begin{tabular}{|c|c|c|c|c|}
\hline Model individual . . . & $\begin{array}{l}\text { Endurance } \\
\text { athlete }\end{array}$ & $\begin{array}{c}\text { Ideal } \\
\text { individual }\end{array}$ & $\begin{array}{l}\text { Moderately } \\
\text { obese }\end{array}$ & $\begin{array}{c}\text { Grossly } \\
\text { obese }\end{array}$ \\
\hline Lean body-wt $(\mathrm{kg})$ & 60 & 60 & 60 & 60 \\
\hline Fat wt $(\mathrm{kg})$ & 5 & 10 & 20 & 30 \\
\hline Total body-wt $(\mathrm{kg})$ & 65 & 70 & 80 & 90 \\
\hline Adiposity $(\%)$ & 8 & 14 & 25 & 33 \\
\hline Fat energy content $(\mathrm{MJ})^{*}$ & 165 & 330 & 660 & 990 \\
\hline Fitness $\dagger$ & High & Medium & Moderate & Poor \\
\hline Reasonable exercise bout $(\mathrm{h}) \dagger$ & 2 & 1.5 & 1 & 0.5 \\
\hline Reasonable exercise rate $(\mathrm{MJ} / \mathrm{h}) \ddagger$ & $3 \cdot 5$ & 3 & 2 & 1.2 \\
\hline Expenditure/bout $(\mathrm{MJ}) \frac{+}{+}$ & 7 & $4 \cdot 5$ & 2 & 0.6 \\
\hline Fat stores utilized (\%) & $4 \cdot 2$ & 1.36 & $0 \cdot 30$ & 0.06 \\
\hline Time interval to total fat depletion (h)§ & 47 & 110 & 330 & 825 \\
\hline Exercise bouts to achieve ideal wt & - & - & 165 & 1100 \\
\hline
\end{tabular}

* Approximating $1 \mathrm{~kg}$ fat $=33 \mathrm{MJ}$.

+ Arbitrarily assigned.

$¥$ From Howley \& Franks (1986).

$\S$ An index of size of fat store.

\| Practical assessment of amount of exercise required. This unrealistically assumes no improvement in fitness.

overweight relatively unfit individual and (4) a very fat, very unfit person. Basic values for the energy costs of the exercise bout are derived from empirical observations reported in the literature (Howley \& Franks, 1986). Performance and endurance times are speculative. Dietary intake is held constant. Before analysing the values in Table 1 I must emphasize that these calculations are theoretical, derived initially from empirical results, but may deviate considerably from what would actually be observed if this experiment were done in practice. However, I am convinced that the qualitative conclusions are correct. A study by Ballor et al. (1990) supports this position. They were unable to show any difference in weight-loss patterns in dieting obese women undertaking either short bouts of intense, or long bouts of moderate exercise. They concluded that the amount of exercise done was simply insufficient to have a significant effect on fat stores in relation to their massive size.

The first observation I would make concerns the efficiency of fat as an energy store. Even an unusually fit individual, the endurance athlete, has many hours of reserve capacity in his minimal fat stores. In evolutionary terms the high energy density of fat, with its dry storage due to its hydrophobic properties, has great selective benefit for a locomotory species. This storage efficiency becomes a penalty for the slimming obese individual. In relation to rates of energy expenditure which are either physically sustainable, or practical in terms of other social demands upon an individual's time, the energy stores of the obese are truly vast. A single bout of exercise in the obese has a tiny net effect in proportional terms, upon energy stores. Only long sustained effort, not just for days and weeks, but for months and years, will have significant effect. This is very graphically illustrated in Table 1.

Obesity has disadvantages for the individual, but for the species genetic variability 
producing a spectrum from lean to obese, could have survival value. In the conditions of prehistory where selective pressures may be presumed still to have been operating and when famine would have been a common occurrence for all mankind, superior fat stores would have potent survival value.

There is one significant way in which Table 1 overstates the case. That is, that with a careful training programme the fat individual can gradually increase both work rate and endurance and, therefore, increase the drain on fat stores at each session. This becomes an accelerating process as weight is lost: but there is a down side to the weight loss, the cost of exercising is reduced. Fortunately it is likely that the benefits of training come earlier than the disbenefits of weight loss. The quantitative relationships between energy expenditure levels at different weights and level of fitness have been tabulated by Howley \& Franks (1986).

\section{REGULATORY COMPENSATION?}

With respect to the energy balance equation (Fig. 1), may it not be that as fuel stores decrease below a central set-point, there is an increased compensatory drive to appetite and feeding? Both human and animal studies substantiate this supposition in individuals whose diet is uncontrolled when physical activity varies. It is difficult in modern studies to obtain naïveté with respect to the energy significance of dietary intake. However, the classic records of Meyer et al. (1956) in a large West Bengal factory make it clear that above a threshold of light activity increasing physical activity demands increasing dietary energy intake for maintenance of body-weight. Below the threshold, however, this relationship appears to break down. However, Garrow (1978) has drawn attention to the fact that the regulatory breakdown below the threshold cannot be unequivocally accepted, allowance must be made for the considerable variance in the collected data: at least one sedentary group (Clerks I) have relatively low body-weight despite an apparently relatively high intake.

\section{REGULATORY RESETTING OF THE 'PONDEROSTAT'?}

It has been suggested that exercise has an input into the setting of the size of body stores. Although such a mechanism may be termed a regulatory resetting of the 'ponderostat' to a lower level, it must be emphasized that the sensory loop (Fig. 1), if such indeed exists, between body stores and hypothalamic appestat has yet to be described. However, there is evidence for such a resetting. Unlike athletes, when sedentary or obese individuals, or both, undertake an exercise programme appetite is not increased. This has been shown in a number of studies reviewed by Wilmore (1983). A regulatory resetting of lower weight with much movement exercise would have advantages in reducing systematically the energy cost, and effort of exercise. Investigation into, and description of, the mechanics of this mechanism is an important outstanding challenge to our full understanding of obesity and the regulation of body-weight.

\section{ADVANTAGES OF EXERCISE SUPPLEMENTING DIETING?}

Dieting is a much more effective and rapid way of bringing about weight loss than exercise alone. However, it is well recognized that there is a diminution of resting 
metabolism in response to what is physiologically starvation, albeit self-imposed. This leads to the situation of 'refractory obesity': the condition when on a substantially reduced diet, because of the compensatory reduction in resting metabolism, a weight plateau is reached at a reduced, but still grossly obese level. This may be due to an active physiological mechanism of defence against starvation to minimize energy consumption (a 'buffer' system, see Garrow (1988)) or a passive event which is simply the consequence of the inevitable loss of lean body mass (Ravussin \& Bogardus, 1989). Shetty (1990) considers that both events occur, first a controlled down-regulation in metabolic rate, second, if lean body mass is lost as dieting continues, whole-body resting metabolism will fall in proportion. This tendency to plateau can be avoided if exercise, at a slowly increasing level as training and fitness allows, adds increasing exercise metabolism to the weight-loss programme, to balance the decreasing resting metabolism and, thus, enable weight loss to continue (Fig. 7-2 of Katch \& McArdle, 1988). Such a programme does work: Reybrouck et al. (1990) for example, have shown that in dieting obese children weight plateaued after a few weeks, whereas the addition of exercise to the programme resulted in a very slow but continuing weight loss sustained for many months.

This role of exercise in enabling weight loss to be sustained in the face of defensive conservation mechanisms, I consider to be the major physiological and psychological advantage of an exercise programme.

\section{EXCESS POST-EXERCISE OXYGEN CONSUMPTION (EPOC)}

Despite what has been said previously, indubitably the occasional individual does lose weight and achieve a clinically 'ideal' body-weight in association with an exercise programme. The weight loss often appears greater than can be expected or explained by the energy cost of the exercise alone. However, rarely if ever has a full quantitative analysis of the energetics of these individuals been undertaken, and dietary restriction may be the main cause of their weight loss. However, it has been supposed from such observations that exercise might be acting not only to consume energy immediately as the fuel for muscular activity, but that it has an indirect effect on whole-body resting metabolism, sustained for some considerable time after the exercise bout itself and, thus, contributing materially to weight loss. I will examine the possibility that this phenomenon of EPOC may significantly contribute to the total energy consumption associated with a bout of exercise: the literature shows contradictory and confusing results when this possibility has been examined experimentally. Values as high as a $12 \%$ stimulation of resting metabolism as long as $12 \mathrm{~h}$ after the exercise bout have been reported (Maehlum et al. 1986). Brehm (1988) has reviewed literature reports investigating EPOC: results vary from as high as $25 \%$, to zero. Just as the variability of the direct effects of exercise on weight loss could be explained by the various and widely different exercise programmes, so could be the case here too. This question has recently been reinvestigated with great care using the best currently available equipment and methodology. These studies were unable to repeat earlier observations showing a major effect of exercise on EPOC. Though EPOC was observed both in obese subjects undergoing an exercise programme (Bingham et al. 1989) and in fit young subjects exercising at various levels (Goldberg et al. 1990; Gore \& Withers, 1990), in no case was the EPOC sufficient to have significant effect on the energy balance equation. However, level and particularly duration of exercise did enhance EPOC (Gore \& Withers, 1990). It 
seems most unlikely that sustained stimulation of resting metabolism after exercise could make a significant contribution to weight loss in the unfit gross obese. Possibly in the mildly obese, sufficiently fit to sustain exercise at a high work rate, EPOC could make a small but significant contribution to weight loss.

\section{FACULTATIVE DIET-INDUCED THERMOGENESIS}

There is a necessary thermic effect of feeding (diet-induced thermogenesis) due to the energy cost of digestion, absorption and metabolic assimilation of the constituents of the meal. It has been suggested that this essential diet-induced thermogenesis may be added to by a facultative element, the degree of which is related to the state of energy reserves, increasing when energy reserves have achieved a regulatory set-point. A failure of this mechanism could lead to obesity (Rothwell \& Stock, 1986). This theory it must be said remains to be proven in man. It has been suggested that exercise before a meal may stimulate the facultative element of diet-induced thermogenesis. Work in this laboratory was able to demonstrate an increase in the thermic effect of feeding in lean individuals, the level of which was related to previous level of exercise (Buckley et al. 1990). However, quantitatively the extra metabolism invoked was insufficient to have any significant effect on the energy balance equation. Preliminary unpublished findings in the obese (P. Ferguson and J. F. Andrews, unpublished results) show no exercise-induced increase in the thermic effect of feeding, hardly surprising at the low levels of exercise of which the obese subjects were capable.

\section{REGIONAL ADIPOSITY: A DIRECT LOCAL EFFECT BY EXERCISE OF ADJACENT MUSCLE MASSES?}

It is commonly held that muscular activity in specific muscle masses will cause loss of fat from associated fat depots: regional exercise affecting regional adiposity, or 'spot' reducing. Many fitness clinics include exercises in their programmes which unquestioningly assume the correctness of this. However, when this question has been investigated by means of an intensive programme of local exercises in several muscle groups (for example leg, Krotkiewski et al. 1979), no effect could be demonstrated on the histology or biochemistry of overlying adipose tissue. Animal studies, however, do show a clear regional effect of exercise (as well as sex differences) in certain adipose tissue depots (Mattocks \& Pond, 1988). This question needs further investigation in man to see if certain regional depots are susceptible to reduction by regional exercise.

\section{BENEFITS AND DANGERS OF EXERCISE IN THE OBESE}

Whatever the effect of exercise, or lack of it, on weight loss it cannot be emphasized too strongly that a programme of exercise at the correct level and of the correct kind must be of benefit in the broad context of improving health and well-being and reducing risk of illness, particularly of cardiovascular disease and diabetes mellitus. In the grossly obese individual or the unfit, or both, or those suffering from cardiovascular disease, only professionally supervised exercise should be undertaken after a previous medical: excessively enthusiastic unsupervised programmes risk joint damage at least, and cardiac damage and even heart failure, at the worst. Exercise has benefits too on blood lipid 
profiles: the high-density lipoprotein:low-density lipoprotein ratio increases (Salonen et al. 1985) with long-term benefit in reducing the risk of cardiovascular disease.

As discussed in the associated review (Prentice et al. 1991) a danger of reduced diets is the loss of protein. A benefit of exercise associated with dieting is that it helps preserve lean body mass (Belko et al. 1987; Stern et al. 1989).

\section{CONCLUSION}

In conclusion, though the direct physical effect of an exercise programme is disappointingly small, the psychological advantages are great: the feeling of increasing well-being as the individual overcomes their unfitness, the fact that the accelerating exercise programme helps to sustain a slow weight loss in the long term, preventing the equilibrium weight which would tend to be attained on a diet alone.

Exercise in moderation is, thus, to be most strongly encouraged, whilst minimizing any false emphasis on its direct effect on adiposity, particularly in the grossly obese.

Much work still needs to be done to define exercise programmes which maximize fatty acid release and their oxidation.

\section{REFERENCES}

Ahlborg, G., Felig, P., Hagenfeldt, L., Hendler, R. \& Wahren, J. (1974). Substrate turnover during prolonged exercise in man: splanchnic and leg metabolism of glucose, free fatty acids and amino acids. Journal of Clinical Investigation 53, 1078-1084.

Ballor, D. L., McCarthy, J. P. \& Wilterdink, E. J. (1990). Exercise intensity does not affect the composition of diet- and exercise-induced body mass loss. American Journal of Clinical Nutrition 51, 142-146.

Belko, A. Z., Van Loan, M., Barbieri, T. F. \& Mayclin, P. (1987). Diet, exercise, weight loss, and energy expenditure in moderately overweight women. International Journal of Obesity 11, 93-104.

Bingham, S. A., Goldberg, G. R., Coward, W. A. \& Prentice, A. M. (1989). The effect of exercise and improved physical fitness on basal metabolic rate. British Journal of Nutrition 61, 155-173.

Björntorp, P. (1982). Interrelation of physical activity and nutrition on obesity. In Diet and Exercise: Synergism in Health Maintenance, p. 91 [P. L. White, editor]. Chicago: American Medical Association.

Brehm, B. A. (1988). Elevation of metabolic rate following exercise. Sports Medicine 6, 72-78.

Buckley, N., Kelly, M., Donne, B. \& Andrews, J. F. (1990). At 30 min post-ingestion the thermic effect of feeding is increased by prior high-intensity exercise. Proceedings of the Nutrition Society 49, 103A.

Epstein, L. H. \& Wing, R. R. (1980). Aerobic exercise and weight. Addictive Behaviours 5, 371-388.

Frayn, K. N., Coppack, S. W., Humphreys, S. M. \& Whyte, P. L. (1989). Metabolic characteristics of human adipose tissue in vivo. Clinical Science 76, 509-516.

Garrow, J. S. (1978). Energy Balance and Obesity in Man, 2nd ed. New York: Elsevier North Holland.

Garrow, J. S. (1988). Obesity and Related Diseases, Chpt 6. Edinburgh: Churchill Livingstone.

Goldberg, G. R., Prentice, A. M., Davies, H. L. \& Murgatroyd, P. R. (1990). Residual effect of graded levels of exercise on metabolic rate. European Journal of Clinical Nutrition 44, 99-105.

Gore, C. J. \& Withers, R. T. (1990). The effect of exercise intensity and duration on the oxygen deficit and excess post-exercise oxygen consumption. European Journal of Applied Physiology 60, 169-174.

Howley, E. T. \& Franks, B. D. (1986). Health/Fitness Instructor's Handbook. Champaign, Ill.: Human Kinetics Publishers.

Katch, F. I. \& McArdle, W. D. (1988). Nutrition, Weight Control, and Exercise. Philadelphia: Lea \& Febinger.

Krotkiewski, M., Aniansson, A., Grimby, G., Björntorp, P. \& Sjostrom, L. (1979). The effect of unilateral isokinetic strength training on local adipose and muscle tissue morphology. European Journal of Applied Physiology 42, 271-281.

Maehlum, S., Grandmontagne, M., Newsholme, E. A. \& Sejersted, O. M. (1986). Magnitude and duration of excess post exercise oxygen consumption in healthy young subjects. Metabolism 35, 425-429. 
Mattocks, C. A. \& Pond, C. M. (1988). Site-specific and sex differences in the rates of fatty acid/triacylglycerol substrate cycling in adipose tissue and muscle of sedentary and exercised dwarf hamsters (Phodopus sungorus). International Journal of Obesity 12, 585-597.

Mattocks, C. A., Sadler, D. \& Pond, C. M. (1987). The effects of exercise and dietary restriction on the activities of hexokinase and phosphofructokinase in superficial, intra-abdominal and intermuscular adipose depots. Comparative Physiology and Biochemistry 87B, 533-542.

Meyer, J., Roy, P. \& Mitra, K. P. (1956). Relation between caloric intake, body weight and physical work: Studies in an industrial male population in West Bengal. American Journal of Clinical Nutrition 4, 169-175.

Newsholme, E. A. (1977). The regulation of intracellular and extracellular fuel supply during sustained exercise. Annals of the New York Academy of Science 301, 81-91.

Prentice, A. M., Goldberg, G. R., Jebb, S. A., Black, A. E., Murgatroyd, P. R. \& Diaz, E. O. (1991). Physiological responses to slimming. Proceedings of the Nutrition Society 50, 441-458.

Ravussin, E. \& Bogardus, C. (1989). Relationship of genetics, age, and physical fitness to daily energy expenditure and fuel utilisation. American Journal of Clinical Nutrition 49, 968-975.

Rennie, M. J., Park, D. M. \& Sulaiman, W. R. (1976). Uptake and release of hormones and metabolites by tissues of exercising leg in man. American Journal of Physiology 231, 967-973.

Reybrouck, T., Vinckx, J., Van Den Berghe, G. \& Vanderschueren-Lodeweyckx, M. (1990). Exercise therapy and hypocaloric diet in the treatment of obese children and adolescents. Acta Paediatrica Scandinavica 79, $84-89$.

Rothwell, N. J. \& Stock, M. J. (1986). Brown adipose tissue and diet-induced thermogenesis. In Brown Adipose Tissue, pp. 269-298 [P. Trayhurn and D. G. Nicholls, editors]. London: Edward Arnold.

Salonen, J. T., Hamynen, H., Leino, U., Kostiainen, E. \& Sahi, T. (1985). Relation of alcohol, physical activity, dietary fat and smoking to serum HDL and total cholesterol in young Finnish men. Scandinavian Journal of Social Medicine 13, 99-102.

Shetty, P. S. (1990). Physiological mechanisms in the adaptive response of metabolic rates to energy restriction. Nutrition Research Reviews 3, 49-74.

Stern, J. S. (1984). Is obesity a disease of inactivity? In Eating and its Disorders, pp. 131-139 [A. J. Stunkard and E. Stellar, editors]. New York: Raven Press.

Stern, J. S., Titchenal, C. A. \& Johnson, P. R. (1989). Obesity: does exercise make a difference? In Obesity in Europe 88. Proceedings of the First European Congress on Obesity, pp. 352-364 [P. Björntorp and S. Rössner, editors]. London and Paris: John Libbey.

Tremblay, A., Despres, J. P. \& Bouchard, C. (1985). The effects of exercise-training on energy balance and adipose tissue morphology and metabolism. Sports Medicine 2, 223-233.

Wallberg-Hendriksson, H. \& Wahren, J. (1989). Effects of nutrition and diabetes mellitus on the regulation of metabolic fuels during exercise. American Journal of Clinical Nutrition 49, 938-943.

Wilmore, J. H. (1983). Body composition in sport and exercise: directions for future research. Medicine and Science in Sports and Exercise 15, 21-31.

Wing, R. R. \& Jeffrey, R. J. (1979). Outpatient treatment of obesity: a comparison of methodology and results. International Journal of Obesity 3, 261-279.

Wolfe, R. R., Klein, S., Carraro, F. \& Weber, J.-M. (1990). Role of triglyceride-fatty acid cycle in controlling fat metabolism in humans during and after exercise. American Journal of Physiology 258, E382-E389. 